\title{
Frontières
}

\section{Les aîné-es trans : une population émergente ayant des besoins spécifiques en soins de santé, en services sociaux et en soins liés au vieillissement}

\author{
Billy Hébert, Line Chamberland et Mickael Chacha Enriquez
}

Volume 25, numéro 1, automne 2012

Le vieillissement et sa diversité

URI : https://id.erudit.org/iderudit/1018231ar

DOI : https://doi.org/10.7202/1018231ar

Aller au sommaire du numéro

Éditeur(s)

Université du Québec à Montréal

ISSN

1916-0976 (numérique)

Découvrir la revue

Citer cet article

Hébert, B., Chamberland, L. \& Enriquez, M. C. (2012). Les aîné-es trans : une population émergente ayant des besoins spécifiques en soins de santé, en services sociaux et en soins liés au vieillissement. Frontières, 25(1), 57-81. https://doi.org/10.7202/1018231ar
Résumé de l'article

Les aîné-es trans sont une population en devenir constituée d'individus aux identités, réalités et trajectoires très diversifiées. Cet article basé sur une recension des écrits, présente tout d'abord cette diversité, notamment en ce qui a trait à l'âge, tant à l'appartenance générationnelle qu'à l'âge du début de la transition. On y traite ensuite de la santé physique des aîné-es trans, soit des problèmes et des besoins de santé qui leur sont propres, puis des barrières auxquelles ils et elles se heurtent dans leurs démarches pour avoir accès à des soins et des services de santé adéquats. Le texte relève certaines difficultés comme l'isolement et le manque de soutien qui sont souvent le lot des aînés trans ainsi que les obstacles dans leur accès aux services sociaux et aux soins liés au vieillissement. L'article propose des pistes d'action pour les personnes professionnelles dans le domaine de la santé et des services sociaux et se conclut sur des pistes de recherche. 


\section{LES AÎNÉ-ES TRANS : UNE POPULATION ÉMERGENTE AYANT DES BESOINS SPÉCIFIQUES EN SOINS DE SANTÉ, EN SERVICES SOCIAUX ET EN SOINS LIÉS AU VIEILLISSEMENT.}

Billy Hébert, M.A.
Chercheur communautaire, Aide aux transsexuel-les du Québec

Line Chamberland, Ph.D.

Professeure, Département de sexologie, Université du Québec à Montréal

Mickael Chacha Enriquez

Candidat à la maîtrise, Département de sociologie, Institut de recherches et d'études féministes, Université du Québec à Montréal

\section{RÉSUMÉ}

Les aîné-es trans sont une population en devenir constituée d'individus aux identités, réalités et trajectoires très diversifiées. Cet article basé sur une recension des écrits, présente tout d'abord cette diversité, notamment en ce qui a trait à l'âge, tant à l'appartenance générationnelle qu'à l'âge du début de la transition. On y traite ensuite de la santé physique des aîné-es trans, soit des problèmes et des besoins de santé qui leur sont propres, puis des barrières auxquelles ils et elles se heurtent dans leurs démarches pour avoir accès à des soins et des services de santé adéquats. Le texte relève certaines difficultés comme l'isolement et le manque de soutien qui sont 
souvent le lot des aînés trans ainsi que les obstacles dans leur accès aux services sociaux et aux soins liés au vieillissement. L'article propose des pistes d'action pour les personnes professionnelles dans le domaine de la santé et des services sociaux et se conclut sur des pistes de recherche.

\section{ABSTRACT}

Trans elders are an emerging population that includes individuals with diverse identities, realities, and life paths. In this literature review, the authors first address this diversity, in particular in regards to one's generation and to the age at which one has begun transition. The authors then discuss trans elders' physical health, with a focus on the specific issues and needs such people can have in regards to health care services, and on the barriers jeopardizing their access to adequate care and services. The isolation and lack of support that many trans elders face is then presented and some of the barriers that exist in accessing social services and age-related care are introduced. The article also offers multiple recommendations for health care and social services providers and concludes with future avenues of research.

MOTS CLÉS : transidentités - transsexualité - transgenre - vieillissement - soins et services de santé - services sociaux

KEYWORDS: transidentities - transsexuality - trangender - aging health care - social services

En 2002, Loree Cook-Daniels, qui a elle-même longuement écrit sur le sujet, soulignait qu'il devenait indispensable de se pencher sur le vieillissement des personnes trans car cette population émergente allait croître dans les années à venir. En 2004, Tarynn M. Witten et Stephen Whittle ajoutaient qu'en plus d'être une population en devenir, les aîné-es trans constituaient un groupe d'individus aux identités, réalités et trajectoires très diversifiées, de sorte que leurs besoins l'étaient également. Witten et Evan Eyler (2006) expliquent d'ailleurs qu'en plus de constituer une population croissante et d'être exposées aux mêmes 
difficultés que toutes les personnes âgées (baisse du revenu, détérioration de la santé, etc.), les personnes aînées trans risquent fort de subir les conséquences de la transphobie. Celle-ci se définit comme la peur, la haine et les préjugés à l'endroit des personnes trans (ASTT(e)Q, 2011 , p. 12), lesquels peuvent se manifester sous forme de violence et de mauvais traitements de la part d'individus, d'organismes ou d'institutions entières (Witten, 2009). Étudier l'intersection du vieillissement et de la transidentité est d'autant plus important que d'autres facteurs de discrimination peuvent s'ajouter - comme l'identité ethnoculturelle, le statut d'immigrant ou la séropositivité.

Cet article propose une synthèse des thèmes abordés par les rares auteurs et auteures qui se sont intéressés à la question du vieillissement chez les personnes trans ${ }^{1}$. L'expression «personnes trans», ou «personnes transidentifiées», englobe un large éventail d'identités revendiquées par, ou attribuées à des personnes dont l'identité de genre ne correspond pas, ou du moins pas exclusivement, au sexe qu'on leur a assigné à la naissance. Il importe de le souligner, la plupart des études recensées ici ne sont malheureusement pas étayées par des données empiriques rigoureuses; il s'agit essentiellement d'extrapolations basées sur ce que l'on connaît du vieillissement dans les populations cis (non trans) ou dans les populations trans tous âges confondus. De plus, ces sources font généralement état seulement des difficultés vécues par les aîné-es trans et ne se penchent donc pas sur leur résilience, ni sur leurs stratégies pour contrer l'adversité.

Dans cet article, nous abordons tout d'abord la diversité de la population des aîné-es trans, notamment en ce qui a trait aux multiples identités pouvant y être incluses. Nous abordons aussi la diversité de cette population quant à l'âge, tant l'appartenance générationnelle que l'âge du début de la transition, définie comme un processus émotionnel et physique durant lequel une personne se perçoit et est perçue comme changeant ou ayant changé d'identité de genre ${ }^{2}$ (Cook-Daniels, 2002). Nous traitons ensuite de la santé physique des aîné-es trans, soit des problèmes et des besoins de santé qui leur sont propres, puis des barrières - en particulier, les diverses formes de discrimination auxquelles ils et elles se heurtent dans leurs démarches pour avoir accès à des soins et des services de santé adéquats. Nous discutons ensuite du bien-être individuel et social des aîné-es trans, en particulier de l'isolement et du manque de soutien qui sont souvent leur lot, 
et nous présentons d'autres difficultés qui entravent leur accès aux services sociaux et aux soins liés au vieillissement. En plus des pistes d'action pour les professionnel-les de la santé et des services sociaux présentes tout au long de l'article, la conclusion propose des pistes de recherche pour les chercheures et les chercheurs qui désirent travailler avec les aîné-es trans.

\section{LA DIVERSITÉ CHEZ LES AÎNÉ-ES TRANS}

Pour aborder la diversité de la population d'aîné-es trans, il faut tenir compte des différentes identités qui peuvent y coexister. Dans la littérature médicale et psychiatrique, le terme «transsexuel-le» fait référence aux personnes qui vivent à temps plein l'appartenance à un sexe autre que celui qu'on leur a assigné à la naissance. Ces personnes peuvent ou non avoir eu recours aux hormones ou à la chirurgie de réassignation de sexe (Cook-Daniels, 2002; ASTT(e)Q, 2011; Witten et al., 2003; Kidd et al., 2008), bien que dans certains milieux, le terme désigne exclusivement les personnes qui ont eu recours à ces étapes de la transition médicale (Witten, 2003). Le terme «transgenre» quant à lui peut être utilisé par une personne qui ne s'identifie ni comme un homme ni comme une femme, ou qui brouille les frontières de genre (Cook-Daniels, 2002; Kidd et al., 2008). Dans certains cas, en particulier dans la littérature médicale et psychiatrique, ce terme désigne les personnes qui, sans vouloir recourir à la chirurgie de réassignation de sexe, prennent des hormones pour vivre plus facilement leur identité de genre (Witten et al., 2003; ASTT(e)Q, 2011). Il y a des auteures et des auteurs qui emploient le terme «transgenre» comme expression parapluie englobant une pluralité de transidentités (Cook-Daniels, 2002 ; Berreth, 2003; Persson, 2009; Kidd et al., 2008; ASTT(e)Q, 2011; Simone et al., 2011; Witten et al., 2004). Dans cet article, nous utilisons les termes «trans» et «transidentités» comme vocables inclusifs, de préférence à «transgenre».

Les sources mentionnées dans cet article portent principalement sur les expériences des personnes transgenres et transsexuelles ayant entamé une transition sociale et médicale. D'autres termes existent pour décrire les identités de personnes pouvant se considérer comme 
transidentifiées. Les travesti-es sont des personnes dont l'identité de genre est généralement conforme au sexe assigné à la naissance, mais qui portent occasionnellement des vêtements culturellement associés à «l'autre sexe» (Cook-Daniels, 2002; Witten, 2003) ou qui endossent «à temps plein ou partiel une expression de genre contraire au sexe assigné à la naissance» (ASTT(e)Q, 2011, p. 10). Le terme «genderqueer» est utilisé, particulièrement dans les communautés anglophones, par des personnes dont l'identité de genre n'est pas binaire - soit qu'elles ne s'identifient pas exclusivement à un sexe (Corwin, 2009), soit qu'elles ne s'identifient à aucun des deux sexes, soit qu'elles s'identifient temporairement à l'un ou à l'autre ou à l'un et l'autre en alternance. Quant au terme «bispirituel» (two-spirited), il est «utilisé dans certaines communautés autochtones nord-américaines pour désigner une personne qui incarne des caractéristiques et qualités à la fois masculines et féminines» (ASTT(e)Q, 2011, p. 11). Ce terme est «aussi utilisé, dans certains contextes autochtones, pour désigner, de manière générale, les personnes trans» (p. 11). Nous désirons préciser que les personnes bi-spirituelles ne se considèrent pas toutes comme trans et que l'application de termes identitaires occidentaux aux communautés autochtones doit se faire sous réserve. Enfin, le terme «cis» (ou cissexuel-le ou cisgenre) est «utilisé dans certaines communautés pour désigner les personnes qui ne sont pas trans» ou dont l'identité de genre est conforme au sexe assigné à la naissance (ASTT(e)Q, 2011, p. 11).

La diversité chez les aîné-es trans inclut aussi d'autres facteurs au-delà de ces identités. D'abord, le terme «aîné-es» englobe des personnes d'âges divers. Correspondant dans plusieurs pays occidentaux, incluant le Canada, à l'admissibilité à la pension de retraite, l'âge de 65 ans est souvent un critère employé pour parler du seuil de la vieillesse, bien que la référence à cet âge précis soit débattue, comme le mentionnent Grenier et Ferrer (2010). En effet, selon Grenier et Ferrer, «le fait d'être considéré comme vieux est en partie déterminé et maintenu par des interactions, des normes et des attentes sociales» (p. 36). Lorsque l'âge chronologique est considéré comme critère de classification, plutôt que l'âge «physiologique» ou encore les étapes de vie franchies, les personnes de 65 ans et plus sont généralement divisées en deux groupes faisant face à des attentes sociales et des présomptions très

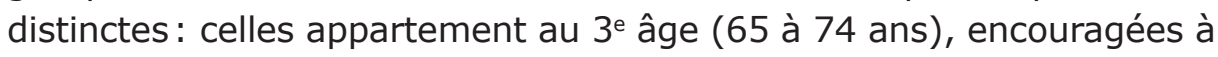
rester actives, et celles du $4^{\mathrm{e}}$ âge ( 75 ans et plus, bien que certains 
mettent la barre à 80 ans), dont les capacités sont souvent considérées comme étant en diminution notable. Bien que Cook-Daniels (2001) n'utilise pas ces standards gérontologiques afin de parler des différents groupes d'âge inclus dans la catégorie des aîné-es trans, l'auteure met l'accent sur les étapes que peuvent vivre ces personnes à différents moments de leur vie (comme le départ des enfants de la maison familiale, la retraite, le déclin de la santé, la préparation aux derniers jours, etc.) et note que les personnes de moins de 65 ans peuvent aussi vivre plusieurs changements liés à l'avancée en âge. Il pourrait donc être plus adapté, comme le suggèrent Grenier et Ferrer (2010), de «faire appel à la perspective du parcours de vie pour nous aider à tenir compte de la diversité» ( $p .50$ ) des cheminements, plutôt que de se fier à l'âge chronologique seulement.

Dans cette veine, selon Persson (2009), les inquiétudes des aîné-es trans sont essentiellement les mêmes que celles de la plupart des personnes vieillissantes (la solitude, la santé et le revenu), mais des problèmes particuliers s'y ajoutent, comme la peur du rejet par la famille, la transphobie des communautés gaies et lesbiennes, et la discrimination exercée contre eux par les personnes professionnelles du domaine de la santé et des services sociaux. Outre ces différences d'expériences entre les aîné-es trans et les aîné-es cis, on distingue aussi deux catégories d'aîné-es trans selon leur parcours : ceux et celles qui ont amorcé leur transition tard dans la vie et ceux et celles qui l'ont entamée plus tôt. Comme le fait remarquer Cook-Daniels (2001), une personne trans qui commence une transition sociale ou médicale à 63 ans a vécu et vivra des expériences très différentes, tant sur le plan de l'adaptation psychologique et physiologique que de l'acceptation sociale, de celles d'une personne aînée trans du même âge qui a effectué sa transition 25 ans plus tôt. Les personnes ayant amorcé leur transition à un plus jeune âge - et qui ont donc passé une bonne partie de leur vie à assumer les divers aspects d'une transition - risquent d'avoir accumulé les expériences négatives et les facteurs de stress (notamment par rapport à leur statut socioéconomique), en plus, dans certains cas, d'avoir reçu une hormonothérapie prolongée (Witten, 2002). Enfin, certaines personnes trans ont eu recours à l'hormonothérapie et à la chirurgie de réassignation de sexe, et d'autres non (tantôt par choix et tantôt parce qu'elles n'y ont pas eu accès) (Witten et al., 2006). 
Plusieurs raisons peuvent amener une personne à faire une transition tardive, notamment la retraite, le départ des enfants de la maison familiale, le décès des parents, une crise médicale ou tout simplement le refus de continuer à se conformer à la pression d'être «un homme masculin ou une femme féminine» (Cook-Daniels, 2002, p. 5). Un âge avancé peut faciliter certains aspects de la transition, puisque les hommes et les femmes ont davantage de caractéristiques physiques en commun en vieillissant (Witten et al., 2003; Witten, 2002). Par contre, plusieurs difficultés peuvent aussi accompagner la transition tardive. Selon Cook-Daniels (2001), les rôles sociaux seraient plus rigides chez les générations plus âgées, tant pour ce qui est des relations interpersonnelles que de la communication verbale et non verbale. Cependant, d'autres auteures et auteurs soutiennent que «les normes [sociales] sont plus clairement spécifiées et plus fortement mises en valeur chez le sujet jeune que chez la personne plus avancée en âge » (Maddox, 2002, p. 54) et de façon plus large, les rôles sociaux sont souvent élargis avec le vieillissement. La nature publique de certains aspects de la transition peut aussi amener des changements considérables pour l'entourage des aîné-es trans - entre autres, pour les partenaires affectifs ou amoureux de longue date, pour les enfants devenus adultes (Sargeant, 2009) - ce qui peut entraîner plusieurs situations de crise dans la vie des personnes trans amorçant leur transition plus tard dans la vie. Il y a des auteures et des auteurs qui avancent que, du moins de nos jours, les hommes trans ${ }^{3}$ semblent $s^{\prime}$ identifier comme trans plus tôt dans la vie, souvent après avoir vécu sous une identité lesbienne; les femmes trans ${ }^{4}$ font souvent une transition plus tardive, parfois après avoir vécu pendant des décennies une vie d'homme «traditionnelle» (Witten et al., 2006; Witten, 2002), ce qui montre l'importance de considérer la diversité des vécus prétransition. Raewyn Connell (2010) donne l'exemple d'une femme trans de 67 ans qui a amorcé une transition sociale et médicale après avoir vécu une vie conventionnelle, exercé des métiers dits masculins et fondé une famille avec une femme. Compte tenu de ce qui précède, il est essentiel que les professionnel-les et chercheur-es saisissent qu'il y a bien des façons d'être un ou une aîné-e trans (Witten et al., 2006; Witten et al., 2003). 


\section{LA SANTÉ DES AÎNÉ-ES TRANS}

À ce jour, on a mené très peu d'études sur les effets de l'hormonothérapie à long terme chez les personnes trans ou de I'hormonothérapie entreprise tardivement chez les aîné-es trans et sur les conséquences de l'interaction entre l'hormonothérapie et le processus normal de vieillissement sur les plans psychologique, physiologique et biomédical (Kidd et al., 2008; Witten, 2002). Malgré ces lacunes, Witten et Eyler (2006) affirment que «les aîné-es trans vivent non seulement les problèmes normatifs du vieillissement, mais aussi, à cause des hormones et autres interventions de réassignation de sexe comme la chirurgie, les problèmes qui résultent du conflit entre ces changements et les processus de vieillissement normatifs» (p. 3) ${ }^{5}$. D'autres auteures et auteurs tendent à corroborer ce constat (Witten, 2009; Witten et al., 2007; Witten et al., 2006; Berreth, 2003; Witten, 2003; Witten et al., 2003; Cook-Daniels, 2002).

Dans Trans Elder Health Issues - sa recension des écrits sur la santé des aîné-es publiée par le Transgender Aging Network (TAN), CookDaniels (2008) mentionne plusieurs problèmes de santé qui semblent être associés à I'hormonothérapie (surtout à long terme) et s'aggraver ou devenir plus fréquents avec l'âge, comme les maladies cardiovasculaires chez les hommes trans, le trombo-embolisme chez les femmes trans, de même que certains types de cancer chez les hommes et les femmes trans. Plusieurs des problèmes de santé liés à I'hormonothérapie deviennent plus fréquents avec l'âge dans la population générale (Witten et al., 2007; Berreth, 2003). Comme l'écrit Berreth (2003), «chez les personnes trans, le risque de souffrir de [certains] problèmes médicaux est accru par les effets conjugués du vieillissement et de I'hormonothérapie. » (p. 47) Par exemple, I'ostéoporose, plus fréquente avec l'âge dans la population générale en raison du déclin hormonal, représente un risque considérable pour les hommes et les femmes trans pour qui il y a eu ablation chirurgicale des gonades (testicules et ovaires) (Cook-Daniels, 2008; Witten et al., 2004). Il semble que des traitements $d$ 'hormonothérapie inadéquats pourraient aussi nuire à la santé buccale, cardiopulmonaire et cardiovasculaire des hommes et des femmes trans (Witten et al., 2004; Witten et al., 2003).

Selon Cook-Daniels (2002), ces risques croisés pourraient aussi empêcher des personnes trans d'accéder à certaines étapes de la transition 
médicale, en particulier les interventions chirurgicales de réassignation de sexe, car plus l'âge avance, plus la personne est susceptible de connaître des problèmes de santé (chroniques ou non) qui peuvent rendre les interventions chirurgicales (de réassignation de sexe ou autres) plus dangereuses (Cook-Daniels, 2008) ou moins accessibles (Witten et al., 2007; Witten et al., 2004). Aussi, de façon générale, en plus des risques associés à ces problèmes de santé, les personnes plus âgées récupèrent moins rapidement après des interventions chirurgicales, en partie parce que les tissus humains perdent leur souplesse en vieillissant (Cook-Daniels, 2008). Selon Witten et Eyler (2007), que I'intervention chirurgicale ait lieu loin ou près du domicile des candidates et des candidats, il faut toujours évaluer soigneusement l'appui des proches et l'accès à d'autres ressources de soutien, car la durée du rétablissement, et donc les besoins de soins postopératoires, augmentent avec l'âge. Compte tenu de tous ces risques, les aîné-es trans doivent passer les tests de dépistage appropriés, notamment un examen de la prostate pour les femmes trans, un examen gynécologique pour les hommes trans et une mammographie pour les hommes et les femmes (Persson, 2009; Berreth, 2003). Ces examens importants devraient s'accompagner d'un suivi médical rigoureux et, chez les personnes sous hormonothérapie, de tests sanguins réguliers (Cook-Daniels, 2008). Selon Persson (2009), d'autres facteurs tels que le «passé chirurgical», le sexe légal et l'incompréhension du personnel médical peuvent rendre difficile l'accès à certains de ces tests.

\section{DISCRIMINATION ET BARRIÈRES À L'ACCÈS AUX SOINS DE SANTÉ}

Les personnes trans âgées se heurtent souvent à l'incompréhension et à la discrimination dans leurs rapports avec le personnel professionnel de la santé. Ceux et celles ayant entamé leur transition il y a des années risquent d'avoir vécu ces problèmes encore plus fréquemment (Cook-Daniels, 2008; Berreth, 2003; Minter, 2003; Witten, 2003; Witten et al., 2003; Cook-Daniels, 2002; Donovan, 2002). Par ailleurs, les personnes aînées qui veulent amorcer une transition tardive hésitent à consulter des professionnel-les de la santé à ce sujet, de crainte de ne pas recevoir des soins adéquats, d'être ridiculisées ou même de subir certaines formes de violence (Witten et al., 2006). 
Comme les personnes trans doivent souvent continuer à consulter régulièrement au moins un médecin une fois la transition «terminée ${ }^{6}$, il importe que ce dernier ou cette dernière soit sensibilisé à leur réalité et à leurs besoins. Cook-Daniels (2002) explique que cela est également vrai lorsque les soins de santé n'ont rien à voir avec la transition: «Les aîné-es trans sous hormonothérapie craignent qu'une fois confinés dans les établissements de santé, on les prive de leurs hormones. Ces aîné-es peuvent donc hésiter à se faire soigner même lorsque leur vie est en danger» (p. 13).

L'auteure constate aussi que l'inconfort prononcé que certaines personnes trans éprouvent par rapport à des parties de leur corps, en particulier leurs organes génitaux, peut être l'une des raisons qui les empêchent de se faire soigner à tout âge, surtout si les soins ou examens requis concernent une partie «sexuée» du corps (examen de la prostate, examen gynécologique, etc.), et à plus forte raison si elles ne désirent pas dévoiler leur transidentité (Cook-Daniels, 2008). De plus, certaines personnes trans taisent leur transidentité jusqu'à ce que surgisse un problème médical urgent, ou encore consultent des professionnelles ou des professionnels de la santé différents (et qui ignorent leur existence mutuelle) pour des soins réservés à la transition et pour les soins généraux. Dans certains cas où seuls les proches et le médecin traitant sont au courant de leur transidentité, la situation peut devenir précaire lorsqu'un problème médical exige que la personne se dévoile pour recevoir des soins urgents ou être hospitalisée (Witten et al., 2004; Cook-Daniels, 2002; Witten 2002). Witten et Eyler (2006) avancent d'ailleurs que le dévoilement non consensuel de la transidentité d'une personne par une professionnelle ou par un professionnel de la santé à d'autres personnes peut mettre en péril la confiance des patients. Les aîné-es trans qui ont amorcé leur transition il y a longtemps sont souvent d'autant plus réticents à dévoiler leur transidentité aux professionnelles et aux professionnels de la santé puisqu'ils et elles ont dû débuter leur transition dans le cadre des protocoles de soins très stricts qui étaient alors appliqués pour accéder aux interventions médicales souhaitées (Berreth, 2003; Cook-Daniels, 2002).

Witten (2003) affirme même que la rigidité des systèmes de classement biomédicaux reliés au sexe et au genre peut rendre difficile I'accès aux services de santé (associés ou non à la transition) pour les 
personnes trans qui n'entrent pas dans leurs catégories. Plus précisément, à cause du caractère pathologique qu'ils et elles attribuent aux transidentités, certains professionnels et certaines professionnelles les de la santé risquent d'aiguiller les aîné-es trans vers des services de santé mentale inutiles ou inappropriés. Donovan (2002) rapporte un cas extrême de ce type de discrimination: lors d'une visite à I'hôpital pour recevoir des soins nullement liés à sa transition, une aînée trans a non seulement été humiliée par le personnel de cette institution, mais aussi hospitalisée pour plusieurs jours, mise sous sédatifs contre son gré et même attachée à son lit. Comme plusieurs professionnel-les de la santé considèrent les transidentités comme des pathologies, les personnes trans sont souvent considérées comme des «cas psychiatriques», quels que soient leurs besoins réels (Witten et al., 2006; Witten, 2002).

Bien que la plupart des médecins soient aptes à faire le suivi d'hormonothérapie chez les personnes trans, la majorité des soins liés à la transition sont offerts par quelques spécialistes, principalement des endocrinologues. Ces spécialistes requièrent presque tous une lettre de personnes professionnelles en santé mentale, comme des psychologues, des sexologues ou des psychiatres, qui confirment que la personne trans a reçu un diagnostic de Trouble de l'identité de genre (TIG) (ASTT(e)Q, 2011; 2012)7. À I'heure actuelle au Québec, deux lettres de professionnel-les en santé mentale sont requises en plus d'une preuve d'hormonothérapie afin qu'une personne soit considérée comme candidate aux traitements de réassignation sexuelle par les chirurgiennes ou les chirurgiens qui font partie du programme de couverture de la Régie d'assurance maladie du Québec (RAMQ). De plus, les règles du Directeur de l'état civil quant au changement de prénom sur les papiers d'identité nécessitent deux lettres (personne professionnelle en santé mentale et médecin ayant prescrit I'hormonothérapie), alors que le changement de mention de sexe requiert deux lettres de chirurgiens, chirurgiennes ou médecins attestant que la personne a subi une intervention impliquant une modification structurale des organes sexuels (vaginoplastie chez les femmes trans, hystérectomie chez les hommes trans). La citoyenneté canadienne est aussi une condition requise pour ces changements d'état civil. En plus d'être une étape contestée par plusieurs personnes militantes et alliées, I'obtention de lettres de personnes professionnelles en santé mentale et l'obligation d'obtenir certaines modifications physiques pour être admissible au 
changement de prénom et de mention de sexe sur les documents d'identification pourrait avoir des conséquences importantes sur le bien-être psychologique des personnes trans de tous âges. Comme le suggère Winter $(2007$; 2009), la psychiatrisation des personnes contribuerait en effet non seulement à la transphobie mais pourrait aussi être vue en elle-même comme un facteur pathogénique. La médicalisation et la psychiatrisation des identités trans peuvent causer des traumatismes physiques et psychologiques permanents chez certaines personnes trans - en particulier, chez celles qui ont vécu des formes extrêmes de violence et de mauvais traitements psychiatriques liés à leur transidentité (Minter, 2003).

La plupart des études recensées ici proviennent des États-Unis où il n'existe à ce jour aucune couverture universelle d'assurance maladie et où les barrières financières à l'accès aux soins de santé restent donc parmi les plus importantes pour les aîné-es trans (Persson, 2009; Minter, 2003; Witten et al., 2003; Cook-Daniels, 2002). Bien que la situation diffère au Canada, les aspects financiers de la transition peuvent aussi grandement compliquer et même empêcher l'accès des aîné-es trans à des soins de santé adéquats, liés ou non à la transition. Au Canada, où la santé est sous juridiction provinciale, dans certaines provinces, certaines étapes de la transition sont remboursées par les régimes d'assurance maladie. Cependant, comme le mentionne l'Action Santé Travesties et Transsexuelles du Québec (ASTT(e)Q, 2011), «[b]ien qu'au Québec, plusieurs interventions chirurgicales spécifiquement trans soient couvertes par le régime provincial d'assurance maladie, les frais exigés par les professionnel-les de la santé mentale pour une lettre d'évaluation sont souvent prohibitifs» (p. 15). De plus, ces interventions ne sont couvertes que pour les immigrantes reçues et les immigrants reçus. Enfin, les personnes qui ont assumé elles-mêmes les frais de leurs interventions chirurgicales de réassignation de sexe avant qu'elles ne soient couvertes par le gouvernement québécois ne sont pas remboursées.

Witten et Eyler (2007), en particulier, formulent de nombreuses recommandations déduites des quelques études existantes sur la santé des personnes trans et de la littérature gérontologique générale. Ainsi, Witten et Eyler suggèrent de tenir compte à la fois des effets habituels 
de la prise d'hormones chez les personnes cis et des caractéristiques médicales particulières de la personne trans. De façon générale, ils soulignent l'importance pour les professionnel-les de la santé de bien informer leurs patient-es trans, en particulier les plus âgés, des risques associés à I'hormonothérapie et à la chirurgie de réassignation de sexe pour les aider à faire des choix éclairés. Ils recommandent également de prescrire aux personnes trans qui veulent suivre une hormonothérapie la dose minimale requise, en raison des risques associés au surdosage, et préconisent l'administration des hormones par voie transdermique plutôt que par voie orale ou par injection. Pour ce qui est de l'ostéoporose, Witten et Eyler suggèrent que les professionnel-les de la santé encouragent et aident les aîné-es trans à cesser de fumer, à réduire leur consommation d'alcool, à maintenir un poids santé et surtout s'il y a eu une ablation chirurgicale des gonades, mais que leur état de santé leur interdit l'hormonothérapie - à recourir à des traitements alternatifs pour contrer l'ostéoporose.

Le fait que les professionnel-les de la santé manquent trop souvent des connaissances nécessaires pour bien desservir les personnes trans (Persson, 2009; Kidd et al., 2008) et l'insuffisance criante de recherches sur les besoins médicaux de ces dernières (Berreth, 2003) sont les principales barrières à l'accès aux soins de santé des personnes trans. Conséquemment, c'est souvent sur ces dernières que retombe la responsabilité d'éduquer les professionnel-les de la santé et donc la nécessité de se renseigner elles-mêmes en profondeur sur la question (Persson, 2009; Cook-Daniels, 2002). Les aîné-es trans peuvent se lasser de toujours avoir à sensibiliser leurs médecins. Le manque de connaissance des professionnel-les de la santé et la «discordance» entre l'anatomie d'une personne, son identité de genre et la perception qu'ont les autres de son genre peuvent donc rendre les soins de santé plus complexes (Witten et al., 2006; Minter, 2003; Witten et al., 2003) - surtout pour les personnes qui n'ont pas eu recours à la chirurgie de réassignation de sexe. Or, pour leur fournir les soins et les tests de dépistage appropriés, et éviter que le non-dévoilement de la transidentité entraîne d'autres problèmes médicaux, les professionnel-les de la santé doivent bien connaître les besoins particuliers des aîné-es trans (Persson, 2009). 


\section{LE BIEN-ÊTRE INDIVIDUEL \\ ET SOCIAL DES AÎNÉ-ES TRANS}

Le soutien social est un concept clé en gérontologie: la présence d'un réseau de soutien stable au cours du vieillissement est primordiale pour les aîné-es de la population générale (Witten et al., 2003). Elle aurait même une influence majeure sur la qualité de vie à long terme (Witten, 2004). Les aîné-es cis qui ont peu de soutien seraient hospitalisés plus souvent (Witten, 2004; Berreth, 2003). L'isolement social pénalise lourdement les aîné-es trans; le manque de soutien, les difficultés relationnelles et le rejet que vivent ces personnes peuvent venir de la famille, de l'entourage ou de la communauté plus large. Pour ces personnes, constate Witten (2004), «le manque d'interaction sociale [positive] se révèle souvent être l'une des principales sources de difficulté» (p. 207), et ce pour plusieurs raisons.

D'abord, non seulement le réseau de soutien des aîné-es trans est plus restreint que celui de leurs pairs cis, mais leur entourage se compose souvent d'ami-es plutôt que de membres de la famille (Persson, 2009; Berreth, 2003). Les relations familiales peuvent souvent changer après la transition, car il faut en effet redéfinir certains rôles, comme ceux de père, de mère, de grand-père, de grand-mère (Witten et al., 2006; Witten, 2002). Les personnes trans qui ont entamé leur transition plus jeunes peuvent avoir vécu le rejet de la part de certains membres de la famille et même en avoir été exclues depuis de nombreuses années. De plus, les parents des aîné-es trans qui n'ont pas rejeté complètement leurs enfants peuvent tout de même avoir du mal à s'adapter à leur nouvelle identité (Cook-Daniels, 2002). Les aîné-es trans peuvent aussi devoir fournir des soins à une personne plus âgée, comme un de leurs parents, avec qui ils et elles ont des relations conflictuelles en partie à cause de leur transition (Witten et al., 2003). Le cas échéant, les répercussions négatives du rôle très exigeant de «proche aidant» - la dépression, par exemple - peuvent être amplifiées par la non-acceptation de la transidentité de l'aidant-e (Kidd et al., 2008; Witten et al., 2003). Les aîné-es qui font une transition tardive peuvent aussi voir les relations avec leurs enfants changer ou se compliquer (Witten et al., 2003); ces enfants peuvent éprouver du désarroi et même de la détresse (Cook-Daniels, 2002). Comme le rapporte Cook-Daniels (2002), certaines personnes aînées trans se voient refuser l'accès à leurs petits-enfants, bien que les enfants en bas âge 
s'adaptent généralement bien à la transition d'une personne de leur entourage. Des problèmes de dévoilement non consensuel et des situations embarrassantes peuvent aussi émerger; ainsi Cook-Daniels (2002) rapporte le cas d'une jeune femme qui appelle «papa» une dame âgée qu'elle accompagne dans les toilettes des femmes.

Les partenaires de longue date peuvent aussi être très affectés par la transition d'une personne aînée trans (Cook-Daniels, 2002; Witten, 2002). Le Transcience Longitudinal Aging Research Study indique que le taux de divorce est très élevé chez les aîné-es trans (Witten, 2003). De plus, $50 \%$ des répondant-es de ce sondage ont déclaré vivre seuls. Les conjoint-es peuvent vivre des difficultés d'adaptation à la «nouvelle orientation sexuelle» qui leur est imposée et à l'image que donne leur couple depuis la transition de leur partenaire (Witten, 2009 ; Cook-Daniels, 2002), comme cela peut se produire dans le cas d'une femme trans et de son épouse qui, autrefois considérées comme un couple hétérosexuel, sont devenues après la transition un couple de même sexe aux yeux des autres. Witten et Eyler (2007) avancent que cette possible redéfinition du couple et la peur du rejet par son ou sa partenaire peuvent inciter certaines personnes à attendre le décès de leur conjoint ou de leur conjointe pour entamer leur transition. Les couples qui restent ensemble pendant et après la transition d'un-e partenaire peuvent aussi perdre leur sentiment d'appartenance à des groupes sociaux et communautaires. Cook-Daniels (2002) rapporte le cas d'un homme trans et de sa partenaire qui, avant la transition, se considéraient comme un couple de même sexe et qui sont maintenant exclus de milieux sociaux lesbiens. Hors du contexte du couple, le rejet des aîné-es qui font une transition tardive par leurs communautés d'appartenance est apparemment fréquent (Witten, 2009). Nombre d'aîné-es ne se sentiront pas, ou ne se sentiront plus, bien accueillis dans les groupes homosexuels ou dans des contextes hétérosociaux, en particulier au début de la transition (Berreth, 2003). De plus, le réseau social élargi d'une personne aînée trans - collègues de travail, ami-es, voisins, connaissances, employés des commerces fréquentés régulièrement - est souvent témoin de la transition de cette personne, ce qui peut engendrer de la discrimination dans le milieu de vie général (Cook-Daniels, 2002).

D'autre part, les aîné-es trans doivent souvent réapprendre à vivre leur sexualité et, disent plusieurs auteur-es, peuvent avoir beaucoup de 
mal à trouver des partenaires, surtout les femmes trans selon la littérature (Witten et al., 2003; Cook-Daniels, 2002; Donovan, 2002; Witten, 2002). Il importe de prendre en considération le fait que souvent les personnes plus âgées sont moins bien informées sur les pratiques actuelles de sécurisexe et sont donc moins portées à se protéger (Witten, 2009; Cook-Daniels, 2008; Witten et al., 2003). Les aîné-es trans qui amorcent une transition à un âge plus avancé peuvent aussi vivre une «deuxième adolescence» à laquelle ils et elles sont mal préparés (Witten, 2003; Cook-Daniels, 2002). Certaines personnes réapprennent à vivre leur sexualité avec leur nouvelle identité de genre; d'autres retrouvent une sexualité active avec de nouveaux partenaires, et parfois leurs dernières relations sexuelles remontaient à une époque où le VIH était moins courant, autant de facteurs qui peuvent augmenter le risque d'infection (Witten et al., 2007) ${ }^{8}$.

La présence d'un éventail de sources de soutien (famille, ami-es, groupes communautaires et religieux, etc.) est indispensable pour les aîné-es, cis ou trans (Witten, 2002). Selon Berreth (2003), le nombre de personnes avec qui un individu peut discuter ouvertement de problèmes liés à sa transidentité influe positivement sur le niveau d'activité de cette personne et sur sa participation au milieu politique et social plus large, ce qui souligne encore une fois l'importance du soutien et des réseaux sociaux dans le bien-être général des aîné-es trans. Witten (2009) considère qu'avec l'âge, le rejet général et familial ainsi que l'isolement qui en découle entraînent «une augmentation des dépressions, une augmentation de la morbidité et une augmentation de la mortalité» (p. 41). Le rejet, l'isolement et le manque de soutien ont donc des conséquences importantes sur la santé générale et mentale de toute personne.

Tout d'abord, il semble clair selon la littérature que les personnes trans souffrent plus souvent de dépression que les personnes cis (Cook-Daniels, 2008; Berreth, 2003), y compris les personnes cis de minorités sexuelles comme les hommes gais (Cook-Daniels, 2008). Par ailleurs, les personnes trans rapportent plus d'idées suicidaires et de tentatives de suicide que la population générale (Cook-Daniels, 2008; Berreth, 2003), mais les résultats d'une étude ontarienne suggèrent que l'expérience de transphobie et d'autres violences est un facteur contribuant fortement à ces taux (Scalon et al., 2012). La littérature indique que les personnes trans, tous âges confondus, 
rapporteraient aussi de plus hauts taux de consommation de psychotropes et d'abus de substances que le reste de la population (Cook-Daniels, 2008; Berreth, 2003). Par exemple, le rapport du National Transgender Discrimination Survey (NTDS) démontre qu'une plus grande proportion de personnes ayant répondu à leur étude, comparativement à la population générale, rapportent faire l'utilisation de drogues ou d'alcool afin de faire face aux mauvais traitements vécus à cause de leur transidentité (Grant et al., 2011). De plus, cette même étude suggère que certains facteurs, comme faire du travail du sexe ou avoir été victime de violence physique transphobe, sont liés à de plus hauts taux de consommation.

Persson (2009) avance que l'isolement social, la peur du dévoilement et la discrimination en milieu de soins de santé peuvent mener certains aîné-es à négliger leurs besoins. Cook-Daniels et Mumson (2010) corroborent cette affirmation dans leur étude sur la violence et ses effets chez les aîné-es trans: un nombre étonnamment élevé de personnes trans de 50 ans et plus déclarent avoir vécu un ou plusieurs épisodes d'incapacité psychologique ou physique si intense(s), par exemple des moments de dépression profonde, qu'ils ou elles n'étaient plus en mesure de combler leurs besoins de base comme l'alimentation, I'hygiène et la sécurité. D'ailleurs, selon Witten et Whittle (2004), «comme le réseau de soutien social des personnes trans est souvent appauvri, on peut raisonnablement postuler une augmentation significative du nombre de personnes qui tombent dans la catégorie de l'autonégligence» (p. 517).

\section{BARRIÈRES À L'ACCÈS AUX SERVICES SOCIAUX ET AUX SOINS LIÉS AU VIEILLISSEMENT}

En plus des besoins que les aîné-es trans ont en commun avec les aîné-es cis - soins et services à domicile ou en résidence quand il y a perte d'autonomie - , ils et elles ont de multiples besoins de soutien et d'aide psychologique. Malheureusement, ces personnes se heurtent à divers obstacles lorsqu'elles tentent d'accéder à ces services. Premièrement, plusieurs des barrières à l'accès aux soins de santé, notamment le manque de sensibilisation du personnel, se dressent 
devant les aîné-es qui tentent d'accéder aux services sociaux et aux services d'aide psychologique (Cook-Daniels, 2008; Berreth, 2003; Minter, 2003; Witten, 2003; Witten et al., 2003; Cook-Daniels, 2002; Donovan, 2002). Ces obstacles ont de graves répercussions, surtout chez les aîné-es dont la santé décline et dont le milieu de vie va changer. Comme la sécurité financière des personnes retraitées dépend aussi de leur statut socioéconomique tout au long de la vie (Witten et al., 2007), les expériences de discrimination qu'ont vécues les aîné-es trans ayant fait leur transition à un plus jeune âge peuvent aussi influer sur le type d'hébergement auquel ils et elles auront accès. À noter, le travail du sexe étant plus fréquemment pratiqué par les personnes trans, en particulier les femmes trans, que par les personnes de la population générale selon l'étude du NTDS (Grant et al., 2011), il est possible que le vieillissement apporte des changements importants aux conditions de travail et au revenu des travailleur-euses du sexe trans ${ }^{9}$.

Comme le souligne Witten (2009), la littérature en gérontologie démontre l'importance du milieu de vie sur la qualité de vie générale des aîné-es. Les établissements de soins palliatifs et les résidences pour personnes en perte d'autonomie se doivent de répondre aux besoins des personnes dont elles s'occupent, et de respecter leur dignité et leur droit à l'autodétermination. Cependant, en pratique, les préjugés et les attitudes discriminatoires des professionnel-les susceptibles d'accueillir des aîné-es trans peuvent nuire au bien-être de ces personnes, lesquelles risquent de ne pas pouvoir compter sur le soutien de leur famille lorsqu'elles revendiquent le respect de leurs droits. Là encore, que les soins soient offerts à domicile ou en résidence, l'incompréhension du personnel risque d'être encore plus grande si les organes génitaux d'une personne ne correspondent pas à son identité de genre (Persson, 2009; Cook-Daniels, 2002; Witten, 2009). Ces difficultés peuvent se compliquer lorsque les capacités cognitives et intellectuelles d'une personne déclinent avec l'âge (Witten, 2009). Kidd et Witten (2008) rapportent aussi le cas du directeur d'un centre de soins pour personnes en perte d'autonomie déclarant que les transidentités sont «une affaire d'homosexualité» (p. 50) ${ }^{10}$. D'autres professionnel-les questionnés par Kidd et Witten nient qu'il puisse y avoir des personnes trans dans leurs établissements et certains avouent que si cela était, ils ou elles refuseraient de leur fournir des soins et des services. Si on ajoute ces attitudes discriminatoires aux risques élevés de 
pauvreté chez les aîné-es trans, on peut raisonnablement supposer que les soins à domicile et I'hébergement dans un établissement de soins de santé sont d'importantes sources d'inquiétude pour les personnes trans (Witten, 2009).

Dans les dernières années de leur vie, les aîné-es peuvent accorder une grande importance à la spiritualité et ce phénomène peut s'accompagner du désir de s'associer à une Église ou à une communauté religieuse (Kidd et al., 2008). Cependant, la plupart des religions traditionnelles refusent d'accepter les personnes trans et cette discrimination peut même s'étendre à leur famille et à leur entourage (Witten et al., 2003). Comme le notent Kidd et Witten (2008) dans leur étude sur la religiosité des hommes âgés trans, il semble aussi que les croyances religieuses des aîné-es trans diffèrent grandement de celles, plus normatives, du reste de la population, ce qui suggère que les aîné-es trans ne se reconnaissent pas non plus dans les grandes religions (par exemple le christianisme, le judaïsme et l'islam). Cela peut signifier que les aîné-es trans, du moins les hommes trans, pourraient faire preuve de créativité afin de réinterpréter les croyances et les normes promues par les grandes religions, afin de réconcilier leur foi à leur identité. En revanche, cela peut aussi signifier que les aîné-es trans se retrouvent une fois de plus isolés, ne trouvant aucune communauté d'appartenance spirituelle adaptée à leurs croyances.

Quelques auteur-es formulent des recommandations pour faciliter I'accès des aîné-es trans aux services de santé et aux services sociaux. Witten (2002) soutient que les gestionnaires de cas en soins gériatriques et les travailleurs sociaux et travailleuses sociales doivent, en plus de leurs autres responsabilités, être à l'écoute de leurs clients trans, s'informer de leurs besoins spécifiques et leur offrir un soutien psychologique. Cela fait, ils et elles seront en mesure de bien conseiller les aîné-es trans sur les soins de santé et les examens de routine nécessaires, et de devenir leurs allié-es auprès des autres personnes professionnelles, comme ceux et celles qui travaillent dans les résidences pour personnes en perte d'autonomie. Witten et Eyler (2006) ajoutent que les intervenantes et les intervenants des services sociaux doivent connaître les personnes professionnelles en soins de santé et en services sociaux déjà sensibilisées pour pouvoir aiguiller les aîné-es trans vers eux et elles. Enfin, ces auteur-es recommandent la création de 
groupes d'information et de soutien pour les aîné-es trans, ainsi que la sensibilisation des groupes communautaires et religieux qui desservent l'ensemble des aîné-es (Witten et al., 2006; Witten, 2002). Cook-Daniels (2002) suggère aux organismes qui disent offrir des services aux minorités sexuelles ou aux populations LGBT de s'assurer qu'ils sont en mesure de bien desservir les clients trans de tous les âges et de s'informer sur les services et organismes qui rejoignent les personnes trans.

\section{DISCUSSION ET PISTES DE RECHERCHE}

Comme le souligne Witten (2003), les multiples facteurs liés au bien-être des aîné-es trans - y compris la santé générale et l'accès aux soins médicaux, le soutien de l'entourage et le milieu de vie, ainsi que la sécurité personnelle et la protection contre la violence - ne devraient pas être considérés comme des éléments distincts, mais plutôt comme des aspects interdépendants du bien-être global de ces personnes. Des recherches qualitatives et, si possible, quantitatives de plus grande envergure seront nécessaires pour mieux connaître les multiples besoins des aîné-es trans et pour rendre visibles leurs expériences et leurs inquiétudes. Ainsi, selon Persson (2009), il devrait y avoir davantage d'études sur les effets à long terme des interventions chirurgicales et de I'hormonothérapie liées à la transition pour bien comprendre leur interaction avec le processus normal de vieillissement. Par ailleurs, les effets des mauvais traitements et du rejet doivent aussi être analysés, de même que les dynamiques familiales et les réseaux sociaux. Les intersections peuvent être difficiles à cerner entre toutes ces variables, mais il est néanmoins nécessaire de dresser un portrait des expériences subjectives des aîné-es trans dans toute leur diversité.

Aucune recherche canadienne sur les aîné-es trans n'a été conduite à ce jour, ce qui dénote le besoin de réaliser des études sur la situation des aîné-es trans dans les contextes canadiens et québécois. La plupart des études recensées ici ne traitent que des difficultés des aîné-es trans et ne nous apprennent rien sur ceux et celles qui réussissent à bien vivre. Ces études ne s'attardent ni à leur résilience ni à leurs stratégies pour vaincre l'adversité. Pour Witten (2009), il serait important 
d'étudier les stratégies d'adaptation des aîné-es trans quant à l'isolement. Il se peut qu'Internet, par exemple, soit une source de soutien et d'information pour les personnes trans âgées; cependant, bon nombre n'ont peut-être pas les ressources nécessaires pour avoir accès à un ordinateur ou les connaissances requises pour s'en servir. Les réseaux sociaux tissés entre aîné-es trans, via Internet ou par d'autres voies, constituent aussi une piste de recherche intéressante afin d'identifier les moyens dont ils et elles disposent pour obtenir de l'information favorisant leur bien-être et pour entrer en contact avec des professionnel-les reconnus comme étant sensibilisés à leurs réalités. Selon Persson (2009), de façon générale, la recherche sur les aîné-es trans devrait produire des données pour guider des initiatives d'éducation, de sensibilisation, de développement de services et de défense des droits. Witten et Eyler (2003) avancent que la recherche doit cerner les problèmes des aîné-es trans, comme le non-respect de leur vie privée et le dévoilement non consensuel, en vue de sensibiliser les professionnel-les de la santé et des services sociaux qui veulent offrir à cette population des services adaptés et respectueux.

Cook-Daniels (2008) ajoute que les professionnel-les de la santé doivent prendre en considération la violence et la discrimination qu'ont vécues et que vivent les personnes trans, car ces facteurs peuvent avoir des répercussions considérables sur leur santé et leur bien-être. Les professionnel-les de la santé devraient aussi se renseigner sur les ressources communautaires vers lesquelles aiguiller les aînés trans. La recherche peut également servir à sensibiliser les responsables des organismes communautaires qui pourraient être en mesure de desservir et conseiller les aîné-es trans, ou du moins de les orienter vers les services appropriés (Persson, 2009; Witten, 2002). Cook-Daniels (2001) ajoute qu'il est important de créer des réseaux de chercheur-es, de professionnel-les de la santé et des services sociaux, et de personnes trans, à l'exemple du Transgender Aging Network (TAN), afin de faciliter la communication entre les divers acteurs qui peuvent contribuer au bien-être global des aîné-es trans. Witten et Eyler (2006) renchérissent en ajoutant que cela devrait être une priorité non seulement pour répondre aux besoins des aîné-es trans, mais aussi pour promouvoir leur pouvoir d'agir (empowerment) et les inclure dans l'élaboration des services qui leur sont destinés. 


\section{BIBLIOGRAPHIE}

ASTT(e)Q (2011). Je m'engage: Un manuel pour les professionnels en santé et services sociaux qui travaillent avec des personnes trans, Montréal, Action Santé Travesties et Transsexuelles du Québec (ASTT(e)Q) et Cactus Montréal.

BERRETH, M. E. (2003). «Nursing care of transgendered older Adults: Implications from the literature», Journal of Gerontological Nursing, vol. $29, n^{\circ} 7$, p. 44-49.

CONNELL, R. (2010). "Two cans of paint: A transsexual life story, with reflections on gender change and history», Sexualities, vol. 13, n० 3, p. 3-19.

COOK-DANIELS, L. (2001). Growing Old Transgender, Milwaukee, FORGE, Transgender Aging Network.

COOK-DANIELS, L. (2002). Transgender Elders and Significant Others, Friends, Family and Allies (SOFFAs): A Primer for Service Providers and Advocates, presented at the 110th Annual Convention of the American Psychological Association, Chicago, Glendale, Transgender Aging Network.

COOK-DANIELS, L. (2008). Trans Elder Health Issues, Milwaukee, FORGE, Transgender Aging Network.

COOK-DANIELS, L. et M. MUMSON (2010). "Sexual violence, elder abuse, and sexuality of transgender adults, age 50+: Results of three surveys», Journal of GLBT Family Studies, vol. 6, n² 2, p. 142-177.

DONOVAN, T. (2002). «Being transgender and older: A first person account», Journal of Gay and Lesbian Social Services, vol. 13, n 4, p. 19-22.

GRANT, J., L.A. MOTTET, J. TANIS, J. HARRISON, J.L. HERMAN et M. KEISLING (2011). Injustice at Every Turn: A Report of the National Transgender Discrimination Survey, Washington, National Center for Transgender Equality and National Gay and Lesbian Task Force.

GRENIER, A. et I. FERRER (2010). «Âge, vieillesse et vieillissement: Définitions controversées de l'âge», dans M. CHARPENTIER, N. GUBERMAN et V. BILETTE (dir.), Vieillir au pluriel. Perspectives sociales, Québec, Les Presses de I'Université du Québec, p. 35-54.

HERBST, J.H., E.D. JACOBS, T.J. FINLAYSON, V.S. MCKLEROY, M. S. NEUMANN et N. CREPAZ (2008). "Estimating HIV prevalence and risk behaviors of transgender persons in the United States: A systematic review», AIDS Behavior, vol. 12, p. 1-17.

KIDD, J. D. et T. M. WITTEN (2008). «Understanding spirituality and religiosity in the transgender community: Implications for aging », Journal of Religion, Spirituality \& Aging, vol. 20, n 1-2, p. 29-62.

MADDOX, G. L. (dir.), (2002). L'encyclopédie du vieillissement, 2ème édition française, Paris, Serdi Edition.

MELENDEZ, R. M., L.A. BONEM et R. SEMBER (2006). «On bodies and research: Transgender issues in health and HIV research articles », Sexuality Research and Social Policy: Journal of NSRC, vol. 3, n' 4, p. 21-38.

MINTER, S. (2003). Legal and Public Policy Issues for Transgender Elder, San Francisco, National Center for Lesbian Rights. 
PERSSON, D. I. (2009). «Unique challenges of transgender aging: Implications from the literature», Journal of Gerontological Social Work, vol. 52, n 6, p. 633-646.

SARGEANT, M. (2009). «Age discrimination, sexual orientation and gender identity: UK/US perspectives», Equal Opportunities International, vol. 28, $\mathrm{n}^{\circ} 8$, p. 634-645.

SCANLON, K., R. TRAVERS, T. COLEMAN, G. BAUER et M. BOYCE (2010). «Ontario's trans communities and suicide: Transphobia is bad for our health», Trans PULSE e-Bulletin, 12 november, 2010, vol. 1, n 2 .

WINTER, S. (2007). Transphobia: a price worth paying for "gender identity disorder"?, communication présentée au Eighteenth Congress du World Association of Sexology, Sydney, Australia.

WINTER, S. (2009). «Cultural considerations for the World Professional Association for Transgender Health's Standards of Care: The Asian perspective», International Journal of Transgenderism, vol.11, n 1, p. 19-41.

WITTEN, T. M. (2002). «Geriatric care and management issues for the transgender and intersex populations», Geriatric Care and Management Journal, vol. 12, n 3, p. 20-24.

WITTEN, T. M. (2003). «Transgender aging: An emerging population and an emerging need», Sexologies, vol. 12, n 44, p. 11-20.

WITTEN, T. M. (2004). «Life course analysis - The courage to search for something more: Middle adulthood issues in the transgender and intersex community », Journal of Human Behavior in the Social Environment, vol. 8, $\mathrm{n}^{\circ} 2$, p. 189-224.

WITTEN, T. M. (2009). «Graceful exits: Intersection of aging, transgender identities, and the family/community», Journal of GLBT Family Studies, vol. 5, n' 1 , p. 33-61.

WITTEN, T. M. et A. E. EYLER (2003). «Transsexuals, transgenders, crossdressers: Issues for professionals in aging - An update», Outword, en ligne. <http://www.asaging.org/LGAIN/outword-063.htm>, consulté le 8 septembre 2011.

WITTEN, T. M. et A. E. EYLER (2006). Transgender Aging: The Graying of Transgender, en ligne. <http://www.people.vcu.edu/ tmwitten/papers/ APHA-Newsletter2006.pdf>, consulté le 13 septembre 2011.

WITTEN, T. M. et A. E. EYLER (2007). «Transgender aging and the care of the elderly transgendered patient», dans R. ETTNER, S. MONSTREY et A.E. EYLER (dir.), Principles of Transgender Medicine and Surgery, New York, Haworth Press, p. 285-310.

WITTEN, T. M. et S. WHITTLE (2004). «TransPanthers: The greying of transgender and the law», Deakin Law Review, vol. 9, n² 2, p. 503-522.

WPATH (2011). Standards of Care for the Health of Transsexual, Transgender, and Gender Nonconforming People, 7th version. The World Professional Association for Transgender Health. 


\section{NOTES}

1 Cet article a été rédigé dans le cadre d'une recherche-intervention financée par le ministère de la Famille et des Aînés dans le cadre du programme Soutien aux initiatives visant le respect des aînés (SIRA). Issu d'un partenariat entre I'Aide aux transsexuel-les du Québec (ATQ) et la Chaire de recherche sur I'homophobie de I'UQAM, ce projet consiste en une recherche communautaire qualitative sur les besoins, les expériences et les craintes des aîné-es trans, en particulier quant à l'accès aux soins de santé, aux services sociaux et aux soins liés au vieillissement. Le volet intervention comprend la conception de matériel d'éducation et de sensibilisation pour les professionnel-les de ces domaines et chez ceux et celles offrant des soins liés au vieillissement. Pour plus d'information, visitez <www.atq1980.org>. Les auteur-es remercient les membres du Comité consultatif du projet pour leurs conseils avisés.

2 Ce processus diffère pour chaque individu et « peut comprendre - ou non - I'adoption d'un nouveau nom, de nouveaux vêtements, de nouveaux prénoms, [et] le recours au traitement hormonal »[ASTT(e)Q, 2011, p. 11] et chirurgical.

3 En anglais parfois sous les formes Female-to-Male ou FTM. Une traduction française pourrait être «femme vers homme».

4 En anglais parfois sous les formes Male-to-Female ou MTF. Une traduction française pourrait être «homme vers femme».

5 Notre traduction. Il en va de même pour les autres citations originalement en anglais.

6 Si certaines personnes cessent I'hormonothérapie ou l'interrompent à un ou plusieurs moments de leur vie, d'autres qui considèrent leur transition terminée vont néanmoins prendre des hormones toute leur vie, ce qui requiert un suivi médical régulier.

7 La World Professionnal Association for Transgender Health (WPATH), une association internationale regroupant des spécialistes en santé trans ainsi que des militants trans, suggère dans la $7^{\mathrm{e}}$ version de ses Standards de soins (WPATH, 2011), son manuel de recommandations pour les professionnel-les offrant des soins aux personnes trans, de s'éloigner du modèle basé sur l'obtention d'un diagnostic. Cet organisme encourage plutôt les intervenant-es en santé à accompagner leurs patient-es dans leur processus de transition, plutôt que d'agir en tant que «gardien-nes des frontières» (gate-keepers). La $5^{\mathrm{e}}$ version du Diagnostic and Statistical Manual of Mental Disorders (DSM-V) de I'American Psychological Association, dont la parution est prévue pour mai 2013, remplacera le diagnostic de Trouble de l'identité de genre pour celui de Dysphorie de genre, auquel s'ajoutera un diagnostic propre aux enfants jugés non conformes aux normes de genre. L'impact de ces nouveaux changements reste à voir pour les personnes trans du Québec qui tenteront d'accéder aux soins de transition. 
8 Pour ce qui est du VIH/SIDA et d'autres infections transmises sexuellement, il est généralement établi que les personnes trans, et en particulier les femmes trans, présentent un risque plus élevé d'infection (Melendez et coll., 2006; Herbst et coll., 2008). Les facteurs qui contribuent à ce phénomène sont multiples et débordent le cadre de cet article, mais en bref, on considère que les relations souvent inégalitaires entre les personnes trans et leurs partenaires, le stigmate social associé à la transidentité et ses lourdes conséquences sur le bien-être, la sécurité et la santé des personnes trans sont les principales causes de la surreprésentation des personnes trans au sein des groupes les plus touchés par le VIH. Plusieurs cofacteurs, comme le fait d'être racisé-e ou d'être travailleur ou travailleuse du sexe, semblent aussi contribuer à ce phénomène.

9 Nous n'avons repéré aucun écrit mettant en lien le travail du sexe chez les personnes trans et le vieillissement.

10 Il est erroné de supposer que les personnes trans sont «non hétérosexuelles», confondant ainsi identité de genre et orientation sexuelle, alors qu'il est généralement admis qu'il s'agit de deux concepts distincts (ASTT(e)Q, 2011). 